\title{
NOVO MÉTODO PARA A QUANTIFICAÇÃO RÁPIDA DE LIPIDIOS EM MICROALGAS
}

\author{
N. N. V. RAMIREZ, C. RANZAN, M. FARENZENA, J. O. TRIERWIELER \\ ${ }^{1}$ Universidade Federal de Rio Grande do Sul, Departamento de Engenharia Química \\ E-mail para contato: \{nelzy, cassiano, farenz, jorge\}@enq.ufrgs.br
}

\begin{abstract}
RESUMO - Lipídios a partir de microalgas têm atraído a atenção de pesquisadores para produção de biocombustíveis. Vários métodos para a quantificação de lipídios neutros, tais como extração com solvente, requerem um grande volume de amostra e tempo. Neste trabalho, Vermelho de Nilo foi utilizado para avaliar o teor de lipídios em microalgas associado à espectroscopia de fluorescência 2D. O cultivo de microalgas foi realizado durante 10 dias com coleta de amostras a partir do $5^{\circ}$ dia de cultivo. $\mathrm{O}$ método padrão utilizado para extração de lipídios foi Bright \& Dyer. A análise utilizada para caracterização com fluorescência consiste em diluir a amostra em etanol ( $\left.40^{\circ} \mathrm{C}, 10 \mathrm{~min}\right)$, e adicionar solução de Vermelho do Nilo $(10 \mu \mathrm{g} / \mathrm{ml}$ em Etanol). Os resultados da quantificação apresentaram $\mathrm{R}^{2}$ de 0,96 , usando modelos lineares selecionados de acordo com a metodologia PSCM. A metodologia proposta apresentou resultados satisfatórios, permitindo medições rápidas e robustas em menos de 15 min.
\end{abstract}

\section{INTRODUÇÃO}

O uso contínuo de combustíveis derivados do petróleo é amplamente reconhecido como insustentável devido ao esgotamento das reservas e à contribuição desses combustíveis para o acúmulo de dióxido de carbono no ambiente. A biomassa de microalga é um excelente candidato como matéria-prima para produção de biodiesel, principalmente devido à alta produtividade, se comparada com matérias-primas convencionais como oleaginosas, gordura animal e óleos usados (Chisti, 2007). Muitas espécies de microalgas apresentam crescimento rápido e produzem diversos tipos de lipídios, triglicerídeos e diglicerídios, fosfolipídios e glicolipídeos, hidrocarbonetos e outros (Hu et al., 2008; Han et al., 2011).

Definir o momento correto para a extração de lipídios a partir de um cultivo é um ponto importante para a otimização do processo, sendo assim necessário o acompanhamento da evolução da concentração de lipídios no decorrer do cultivo. Existem vários métodos para a determinação do teor de lipídios em microalgas. Alguns desses métodos são: cromatografia em camada fina (TLC), HPLC, cromatografia gasosa (GC/MS), cromatografia líquida (LC/MS) e o gravimétrico. O método mais utilizado é o gravimétrico que compreende a extração usando solventes (normalmente orgânicos ou clorados, tais como o hexano ou o clorofórmio) de uma quantidade conhecida de biomassa seca. A maior desvantagem desse método é a necessidade do uso de quantidades relativamente grandes de 


\section{9 a 22 de outubro de 2014 \\ Florianópolis/SC}

amostras e reagentes; além disso, requer demorados processos de secagem e extração (Chen et al., 2011). Para uma quantificação mais eficiente, podem ser usados métodos que envolvem o rompimento da parede celular das microalgas antes da extração dos lipídios (Lee et al., 2010). Assim, é necessário avaliar alternativas mais rápidas de medição, que necessitem de menor quantidade de amostra e que utilizem solventes menos perigosos ao manuseio.

Uma possível solução seria a utilização de um método, rápido e menos agressivo, baseado em espectroscopia de fluorescência. A espectroscopia de fluorescência permite quantificar o teor de lipídios in situ, desde que seja usado um marcador fluorescente apropriado. O Vermelho do Nilo (9dietilamino-5H-benzo $[\alpha]$-fenoxazina-5-ona) tem sido amplamente utilizado como um marcador de lipídios in situ, com a vantagem de possuir uma forte fluorescência em ambientes não-polares, sendo sensível à sua percentagem, permitindo sua quantificação através de medições de fluorescência (Diaz et al., 2008; Chen et al., 2011;De La Hoz Siegler et al., 2012).

O presente estudo tem por objetivo propor e avaliar a viabilidade de um método que permita a quantificação de lipídios da microalga Scenedesmus sp. baseada em medidas de espectroscopia fluorescente. O procedimento proposto embasou-se no protocolo experimental apresentado por Chen et al. (2011) e De La Hoz Siegler et al. (2012), mas com modificações no preparo das amostras e com medidas de intensidade de fluorescência abrangendo um intervalo excitação variando de 300nm à 800nm e emissão de 300nm à 850nm. Modelos quimiométricos baseados em pares de fluorescência selecionados para a caracterização das amostras são ajustados e validados. No caso do método ser viável pode ser utilizado para quantificar os lipídios de forma rápida e sem necessidade de grandes volumes de amostra.

\section{MATERIAIS E METODOS}

\subsection{Preparo do meio de cultivo}

A microalga utilizada neste trabalho é a Scenedesmus sp. O meio de cultivo utilizado foi o Guillard Modificado. Este meio é composto por $10 \mathrm{ml}$ de cada componente do meio Guillard, recomendado por Stein (1979), sem adição de vitaminas.

\subsection{Preparo dos fotobiorreatores}

Foram montados sete fotobiorreatores air-lift de aproximadamente 3 litros cada (para maiores informações ver o trabalho de Gris et al., 2013). Os fotobiorreatores foram mantidos à temperatura de $20^{\circ} \mathrm{C}$, temperatura recomendada por Xin et al. (2011) para obtenção de maior conteúdo de lipídios. O fluxo de ar injetado em cada fotobiorreator foi de $0.75 \mathrm{~L} \cdot \mathrm{min}^{-1}$ e a intensidade luminosa à qual o meio foi exposto foi de 10000 lux $\left(\sim 135 \mu \mathrm{mol} . \mathrm{m}^{-2} . \mathrm{s}^{-1}\right)$. A concentração de biomassa foi determinada através de medidas de densidade óptica para o comprimento de onda de $570 \mathrm{~nm}\left(\mathrm{DO}_{570 \mathrm{~nm}}\right)$, usando espectrofotômetro (UV-1600,Pró-Análise). Os valores de absorbância foram convertidos em concentração de biomassa segundo a equação 1. O tempo de cultivo foi de 10 dias. A Equação 1 apresenta a curva de calibração ajustada para conversão de medidas de densidade óptica em concentração de biomassa. 


\section{9 a 22 de outubro de 2014 \\ Florianópolis/SC}

Biomassa $\left[\right.$ g. L $\left.^{-1}\right]=0,4669 * D_{570 n m}-0,0243$

\subsection{Secagem de amostras de biomassa microalgal}

Como as concentrações de biomassa foram baixas nos primeiros quatro dias, amostras foram coletadas apenas a partir do quinto dia. Diariamente, o conteúdo de um dos reatores foi retirado e centrifugado em tubos de $50 \mathrm{~mL}$. Foram centrifugados 2,5L de meio de cultivo em agitador tipo shaker (modelo MA-563) por $8 \mathrm{~min}$, a $4800 \mathrm{rpm}$ e $4^{\circ} \mathrm{C}$ com tempo de aceleração e frenagem de $30 \mathrm{~s}$. A fim de obter biomassa seca, o conteúdo resultante do processo de centrifugação foi distribuído em vidros relógio, previamente pesados, e levados a estufa de secagem com circulação e renovação de ar (modelo SL102/100). Experimentos prévios mostraram que o tempo de residência ideal das amostras de microalga dentro da estufa, a $40^{\circ} \mathrm{C}$, é de 18 horas.

\subsection{Determinação de conteúdo lipídico}

De posse das amostras de biomassa seca, é dado inicio ao procedimento de determinação dos lipídios, através de dois métodos: espectroscopia de fluorescência e pelo método de Bligh \& Dyer com ultrassom.

Método de espectroscopia de fluorescência: Foram pesados 0,005 g de biomassa seca e esta foi diluída em etanol, em microtubo eppendorf, até completar $1 \mathrm{~mL}$. Posteriormente, foi agitada em vortex e levada a estufa a $40^{\circ} \mathrm{C}$ durante 10 min. Para cada amostra medida no espectrofluorômetro foi preparado $3 \mathrm{~mL}$ de uma solução composta de: $50 \mu \mathrm{L}$ da biomassa diluída, $150 \mu \mathrm{L}$ da solução de Vermelho do Nilo (10 $\mu \mathrm{g} / \mathrm{mL}$ em etanol) e 2,8 $\mathrm{mL}$ de etanol. A concentração final de Vermelho do Nilo foi de $0,5 \mu \mathrm{g} / \mathrm{mL}$, conforme recomendado por Chen et al. (2009). As amostras foram analisadas em espectrofluorômetro (modelo FluoroMax-4 da marca Horiba), e as intensidades foram obtidas a partir de varreduras de excitação de $300 \mathrm{~nm}$ a $800 \mathrm{~nm}$ e emissão de $300 \mathrm{~nm}$ a $850 \mathrm{~nm}$ com incremento de $10 \mathrm{~nm}$. Foi realizado o mesmo procedimento para amostra de $0,05 \mathrm{~g}$. Os dados obtidos foram avaliados de acordo com a metodologia PSCM, proposta por Ranzan et al. (2014), onde modelos quimiométricos, baseados em pares de fluorescência puros, são ajustados através de modelos multilineares. Os pares de fluorescência são selecionados através da utilização do algoritmo de otimização heurístico ACO (Ant Colony Optimization) e combinados de forma a predizer a concentração de lipídios nas amostras. Por este método tratar-se de um método de modelagem assistido, no processo de calibração e ajuste de modelos é necessário que as amostras estejam previamente caracterizadas com relação à concentração de lipídios das amostras do conjunto de calibração.

Método de Bligh \& Dyer com Ultrassom: O método em questão foi adaptado por Gris (2011) para aplicação em cultivos de microalgas. Foram pesados $500 \mathrm{mg}$ da biomassa seca em balança analítica, macerados em cadinho de porcelana com $30 \mathrm{ml}$ de clorofórmio e depois foram adicionados 
$10 \mathrm{ml}$ de metanol à mistura. A mistura resultante foi transferida para um tubo plástico de $50 \mathrm{ml}$ e mantida em banho ultrassom de $40 \mathrm{kHz}$ por $30 \mathrm{~min}$. Após esse tempo, foi adicionado $12 \mathrm{ml}$ de $\mathrm{NaCl}$ $1 \%$ ao tubo e transferiu-se o conteúdo para um funil de separação. Após agitação vigorosa há a separação da solução em duas fases. A fase pesada, correspondente à fase lipídica, deve ser filtrada. O conteúdo lipídico foi transferido para um balão volumétrico de massa conhecida e colocado em um rotaevaporador a $30^{\circ} \mathrm{C}$, por tempo suficiente para a retirada completa de solvente, sendo então transferido a um dessecador até atingir temperatura ambiente. O balão com a amostra resultante é pesado e a quantidade de lipídios obtida por diferença.

\section{RESULTADOS E DISCUSSÃO}

\subsection{Curva de crescimento}

Foi realizada a medição da curva de crescimento na fase exponencial. Através da Figura 1, pode-se observar as medidas de biomassa entre reatores durante 10 dias. Nos últimos dias, o volume reacional apresentou diminuição, devido à retirada de alíquotas amostrais para a quantificação da concentração lipídica através dos dois métodos empregados, fluorescência (necessário 0,055g de biomassa seca por amostra) e qualitativo (0,5g de biomassa seca por amostra).

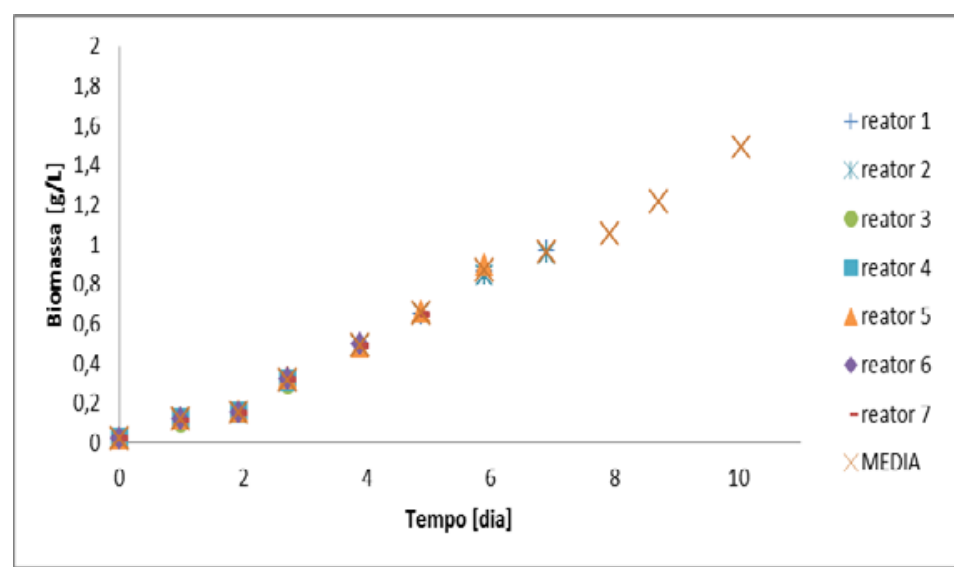

Figura 1 - Curva de crescimento da microalga Scenedesmus sp. em fotobiorreatores air-lift nas condições de $20^{\circ} \mathrm{C}$ e 10000 lux.

\subsection{Conteúdo de lipídios - método tradicional}

A Figura 2 apresenta os resultados da extração de lipídios obtidos pelo método tradicional (Bright \& Dyer). Observa-se que o conteúdo atingiu a máxima concentração no $7^{\circ}$ dia de cultivo, e a porcentagem de lipídios se mantém nos dias seguintes. 


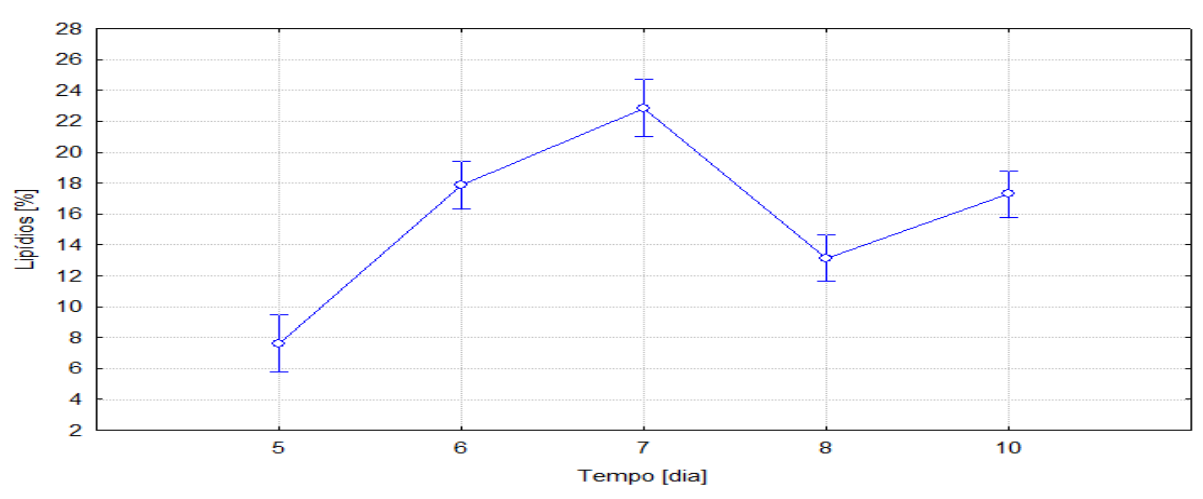

Figura 2 - Conteúdo de lipídios da microalga Scenedesmus sp.

A Figura 3 apresenta os dados de concentração de lipídios [g. $\mathrm{L}^{-1}$ ], para observar o comportamento da quantidade de lipídios ao longo do cultivo. Observa-se que o conteúdo de lipídios aumenta no $7^{\circ}$ dia e depois vai diminuindo ou apresenta valor semelhante. Assim, pode ser útil extrair os lipídios no $7^{\circ}$ dia ou continuar o cultivo para obtenção de maior quantidade de biomassa e avaliação da presença de outros compostos.

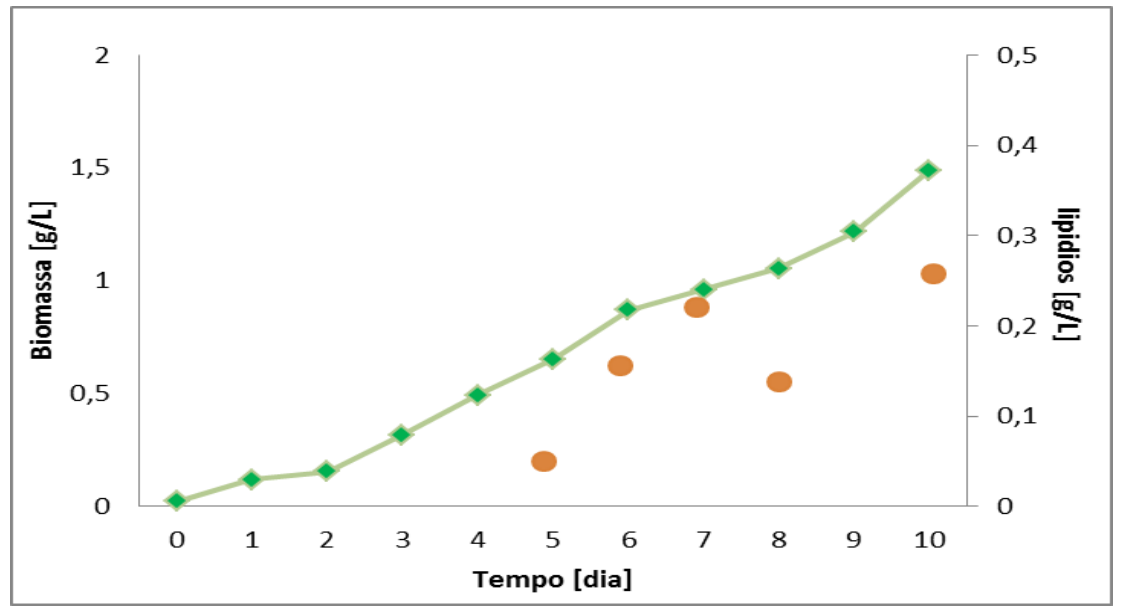

Figura 3 - Quantidade biomassa e lipídios da microalga Scenedesmus sp.

\subsection{Conteúdo de lipídios - Fluorescência}

Os dados obtidos pelas medições de fluorescência foram comparados com os dados obtidos pelo método Bright \& Dyer com ultrassom (vistos na Figura 2). Foram estimados modelos de 1 a 6 pares de excitação/emissão utilizando as intensidades de fluorescência relativas, isto é, as intensidades foram subtraídas da intensidade da amostra de branco do método analítico, sem adição da amostra de microalga, essa diferença visa compensar a influência do meio de cultivo. Na Tabela 1 têm-se os 6 melhores modelos ajustados com os respectivos coeficientes de determinação apresentados por cada um na etapa de calibração. 
Tabela 1 - Pares de excitação/emissão utilizados na obtenção de modelos quimiométricos e resultados de coeficientes de correlação apresentados na etapa de calibração dos mesmos.

\begin{tabular}{|c|c|c|c|c|c|c|c|}
\hline \multicolumn{8}{|c|}{ Diluição 0,05 } \\
\hline $\begin{array}{l}\text { Tamanho } \\
\text { do modelo }\end{array}$ & $\mathbf{R}^{2}$ & \multicolumn{6}{|c|}{ Pares de fluorescência selecionados } \\
\hline 1 & 0,94708 & $\begin{array}{l}\text { Ex640/ } \\
\text { Em670 }\end{array}$ & & & & & \\
\hline 2 & 0,980577 & $\begin{array}{l}\text { Ex620/ } \\
\text { Em690 }\end{array}$ & $\begin{array}{l}\text { Ex640/ } \\
\text { Em740 }\end{array}$ & & & & \\
\hline 3 & 0,989816 & $\begin{array}{l}\text { Ex620/ } \\
\text { Em630 }\end{array}$ & $\begin{array}{l}\text { Ex640/ } \\
\text { Em750 }\end{array}$ & $\begin{array}{l}\text { Ex550/ } \\
\text { Em630 }\end{array}$ & & & \\
\hline 4 & 0,996385 & $\begin{array}{l}\text { Ex340/ } \\
\text { Em400 }\end{array}$ & $\begin{array}{l}\text { Ex730/ } \\
\text { Em790 }\end{array}$ & $\begin{array}{l}\text { Ex430/ } \\
\text { Em800 }\end{array}$ & $\begin{array}{l}\text { Ex610/ } \\
\text { Em620 }\end{array}$ & & \\
\hline 5 & 0,999465 & $\begin{array}{l}\text { Ex300/ } \\
\text { Em780 }\end{array}$ & $\begin{array}{l}\text { Ex370/ } \\
\text { Em780 }\end{array}$ & $\begin{array}{l}\text { Ex650/ } \\
\text { Em790 }\end{array}$ & $\begin{array}{l}\text { Ex400/ } \\
\text { Em620 }\end{array}$ & $\begin{array}{l}\text { Ex430/ } \\
\text { Em470 }\end{array}$ & \\
\hline 6 & 0,999732 & $\begin{array}{l}\text { Ex450/ } \\
\text { Em620 }\end{array}$ & $\begin{array}{l}\text { Ex540/ } \\
\text { Em550 }\end{array}$ & $\begin{array}{l}\text { Ex560/ } \\
\text { Em710 }\end{array}$ & $\begin{array}{l}\text { Ex620/ } \\
\text { Em770 }\end{array}$ & $\begin{array}{l}\text { Ex430/ } \\
\text { Em750 }\end{array}$ & $\begin{array}{l}\text { Ex400/ } \\
\text { Em590 }\end{array}$ \\
\hline \multicolumn{8}{|c|}{ Diluição 0,005 } \\
\hline $\begin{array}{c}\text { Tamanho } \\
\text { do modelo }\end{array}$ & $\mathbf{R}^{2}$ & \multicolumn{6}{|c|}{ Pares de fluorescência selecionados } \\
\hline 1 & 0,943868 & $\begin{array}{l}\text { Ex610/ } \\
\text { Em740 }\end{array}$ & & & & & \\
\hline 2 & 0,986006 & $\begin{array}{l}\text { Ex330/ } \\
\text { Em570 }\end{array}$ & $\begin{array}{l}\text { Ex350/ } \\
\text { Em560 }\end{array}$ & & & & \\
\hline 3 & 0,99377 & $\begin{array}{l}\text { Ex350/ } \\
\text { Em710 }\end{array}$ & $\begin{array}{l}\text { Ex320/ } \\
\text { Em590 }\end{array}$ & $\begin{array}{l}\text { Ex330/ } \\
\text { Em350 }\end{array}$ & & & \\
\hline 4 & 0,996229 & $\begin{array}{l}\text { Ex330/ } \\
\text { Em660 }\end{array}$ & $\begin{array}{l}\text { Ex350/ } \\
\text { Em580 }\end{array}$ & $\begin{array}{l}\text { Ex510/ } \\
\text { Em720 }\end{array}$ & $\begin{array}{l}\text { Ex370/ } \\
\text { Em500 }\end{array}$ & & \\
\hline 5 & 0,998807 & $\begin{array}{l}\text { Ex620/ } \\
\text { Em710 }\end{array}$ & $\begin{array}{l}\text { Ex430/ } \\
\text { Em530 }\end{array}$ & $\begin{array}{l}\text { Ex360/ } \\
\text { Em680 }\end{array}$ & $\begin{array}{l}\text { Ex460/ } \\
\text { Em650 }\end{array}$ & $\begin{array}{l}\text { Ex610/ } \\
\text { Em700 }\end{array}$ & \\
\hline 6 & 0,999789 & $\begin{array}{l}\text { Ex610/ } \\
\text { Em680 }\end{array}$ & $\begin{array}{l}\text { Ex340/ } \\
\text { Em410 }\end{array}$ & $\begin{array}{l}\text { Ex700/ } \\
\text { Em730 }\end{array}$ & $\begin{array}{l}\text { Ex300/ } \\
\text { Em560 }\end{array}$ & $\begin{array}{l}\text { Ex390/ } \\
\text { Em650 }\end{array}$ & $\begin{array}{l}\text { Ex470/ } \\
\text { Em700 }\end{array}$ \\
\hline
\end{tabular}

Por tratar-se de um método assistido, os resultados obtidos pelo método analítico de Bright \& Dyer são utilizados como variáveis de entrada do método proposto para caracterização da concentração lipídica com medidas de fluorescência. Neste processo, dados de fluorescência e respectivas concentrações de lipídios das amostras de microalga são separados em dois conjuntos de dados, um utilizado na etapa de calibração dos modelos, e outro na etapa de testes.

O conjunto de amostras destina à etapa de calibração é composto por 2/3 do numero total de amostras disponível, deixando $1 / 3$ restante para o teste dos modelos gerados. A segmentação 
do conjunto de dados amostral é feita aleatoriamente, empregando a estratégia de validação cruzada no ajuste e teste dos modelos.

A partir dos resultados apresentados na Tabela 2, foram realizados testes para predizer os valores de porcentagem de lipidios das amostras destinadas à etapa de testes, a fim de verificar a validade dos modelos ajustados aos pares determinados.

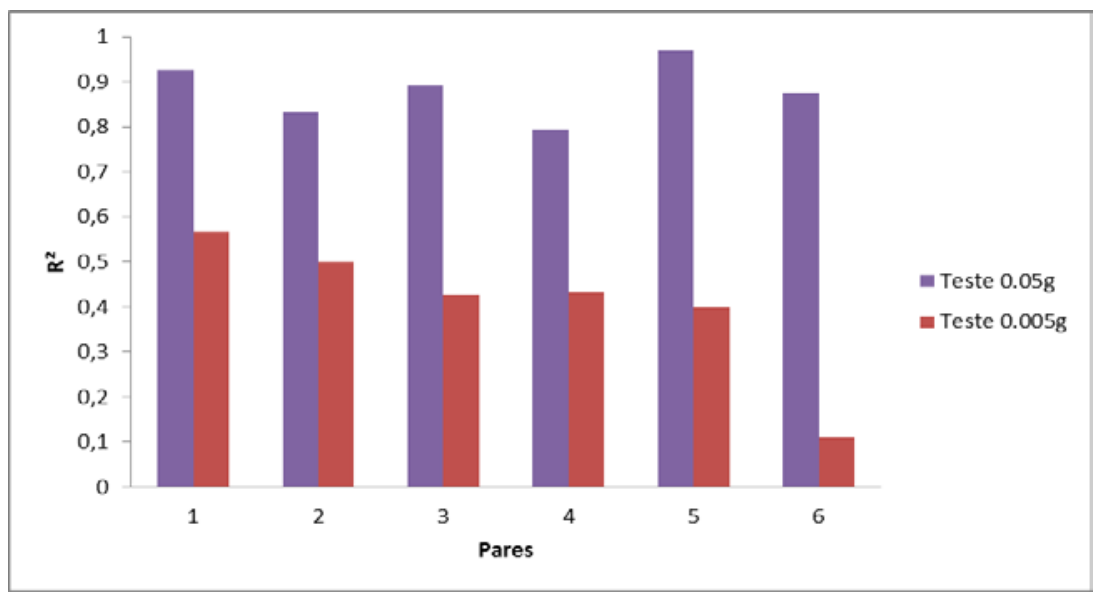

Figura 4 - Coeficiente de correlação de dados preditos pelo número de pares usados.

A Figura 4 apresenta o ceficiente de correlação em funcão do tamanho do modelo ajustado, tanto para a diluição de amostra de $0,05 \mathrm{~g}$ como para $0,005 \mathrm{~g}$. Pode se observar que os melhores resultados foram obtidos nas amostras avaliadas com 0,05g de biomassa. Modelos com 1 e 5 pares apresentaram os melhores resultados de predição, com $\mathrm{R}^{2}$ igual a 0,92 e 0,96, respectivamente. Os resultados apresentados por estes modelos indicam que a metodologia apresenta viabilidade para predizer adequadamente o conteúdo de lipidios. Os resultados mostram que os melhores valores de medida de excitação estão entre 360 a $620 \mathrm{~nm}$ e emissão entre $530 \mathrm{~nm}$ a $740 \mathrm{~nm}$, restringindo a coleta de dados espectrais a um número limitado de pares de fluorescencia.

\section{CONCLUSÕES}

Foi proposto um método de quantificação de lipídios da microalga Scenedesmus sp. baseado em medidas de espectroscopia de fluorescência, comparando dados obtidos pelo método quantitativo com dados obtidos por fluorescência. Conclui-se que é possível usar este método usando amostras com taxa de diluição de 0,05.

Com relação à caracterização da concentração de lipídios em amostras de microalga, o método baseado em medidas de espectroscopia fluorescente apresentou resultados promissores para esta técnica. Os resultados mostram que modelos quimiométricos compostos por 5 pares de fluorescência, e utilizando taxa de diluição de 0,05 , apresentaram $\mathrm{R}^{2}$ superior a 0,9 na etapa de 
testes, confirmando a viabilidade da metodologia de caracterização.

Apesar dos bons resultados de coeficientes de determinação apresentado na etapa de calibração, modelos ajustados usando taxa de diluição de 0,005 não apresentaram resultados significativos na etapa de testes. Este resultado indica que estudos aprofundados devem ser realizados para determinação da taxa de diluição ideal a ser utilizada, tornando o processo de amostragem menos invasivo, e a medição o mais precisa possível.

\section{REFERÊNCIAS}

CHEN, W.; SOMMERFELD, M.; HU, Q. Microwave-assisted Nile red method for in vivo quantification of neutral lipids in microalgae. Bioresource Technology, v. 102, n. 1, p. 135141, 2011. ISSN 0960-8524.

CHISTI, Y. Biodiesel from microalgae. Biotechnology Advances, v. 25, n. 3, p. 294-306, 2007. ISSN 07349750 (ISSN).

DE LA HOZ SIEGLER, $\mathrm{H}$. et al. Improving the reliability of fluorescence-based neutral lipid content measurements in microalgal cultures. Algal Research, v. 1, n. 2, p. 176-184, 10// 2012. ISSN 2211-9264.

DIAZ, G. et al. Hydrophobic characterization of intracellular lipids in situ by Nile Red red/yellow emission ratio. Micron, v. 39, n. 7, p. 819-824, 10,2008. ISSN 0968-4328.

GRIS, L. R. S. Produção da microalga Nannochloropsis oculata em fotobiorreator airlift. 2011. Mestrado em Engenharia Química. Departamento de engenharia química, Universidade Federal do Rio Grande do Sul, Porto Alegre RS Brasil.

GRIS, L. R. S. et al. Laboratory apparatus to evaluate microalgae production. Brazilian Journal of Chemical Engineering, v. 30, p. 487-497, 2013. ISSN 0104-6632.

HAN, Y. et al. Review of Methods Used for Microalgal Lipid-Content Analysis. Energy Procedia, v. 12, n. 0, p. 944-950, // 2011. ISSN 1876-6102.

HU, Q. et al. Microalgal triacylglycerols as feedstocks for biofuel production: perspectives and advances. The Plant Journal, v. 54, n. 4, p. 621-639, 2008. ISSN 1365-313X.

LEE, J.-Y. et al. Comparison of several methods for effective lipid extraction from microalgae. Bioresource Technology, v. 101, n. 1, Supplement, p. S75-S77, 1// 2010. ISSN 0960-8524.

RANZAN, C. et al. Wheat flour characterization using NIR and spectral filter based on Ant Colony Optimization. Chemometrics and Intelligent Laboratory Systems, v. 132, n. 0, p. 133-140, 3/15/ 2014. ISSN 0169-7439.

STEIN, J. R. Handbook of Phycological Methods: Culture Methods and Growth Measurements. Cambridge University, 1979.

XIN, L.; HONG-YING, H.; YU-PING, Z. Growth and lipid accumulation properties of a freshwater microalga Scenedesmus sp. under different cultivation temperature. Bioresource Technology, v. 102, n. 3, p. 3098-3102, 2011. ISSN 09608524 (ISSN). 